\title{
A Particle-Based Model for Effective Properties in Infiltrated Solid Oxide Fuel Cell Electrodes
}

\author{
A. Bertei, ${ }^{a, *, z}$ J. G. Pharoah, ${ }^{\text {b,c,* }}$ D. A. W. Gawel, ${ }^{\text {b,c }}$ and C. Nicolella ${ }^{a}$ \\ ${ }^{a}$ Department of Civil and Industrial Engineering, University of Pisa, 56126 Pisa, Italy \\ ${ }^{b}$ Queen's-RMC Fuel Cell Research Centre, Kingston, Ontario K7L 5L9, Canada \\ ${ }^{c}$ Department of Mechanical and Materials Engineering, Queen's University, Kingston, Ontario K7L 3N6, Canada
}

\begin{abstract}
A modeling framework for the numerical reconstruction of the microstructure of infiltrated electrodes is presented in this study. A particle-based sedimentation algorithm is used to generate the backbone, while a novel packing algorithm is used to randomly infiltrate nanoparticles on the surface of backbone particles. The effective properties, such as the connected triple-phase boundary length, the effective conductivity, the effective diffusivity, are evaluated on the reconstructed electrodes by using geometric analysis, finite volume and random-walk methods, and reported in dimensionless form to provide generality to the results. A parametric study on the effect of the main model and operating parameters is performed. Simulations show that the critical loading (i.e., the percolation threshold) increases as the backbone porosity decreases and the nanoparticle diameter increases. Large triple-phase boundary length, specific surface area and good effective conductivity can be reached by infiltration, without detrimental effects on the effective transport properties in gas phase. Simulations reveal a significant sensitivity to the size and contact angle of infiltrated particles, suggesting that the preparation process of infiltrated electrodes should be properly tailored in order to obtain the optimized structures predicted by the model.

(C) The Author(s) 2014. Published by ECS. This is an open access article distributed under the terms of the Creative Commons Attribution Non-Commercial No Derivatives 4.0 License (CC BY-NC-ND, http://creativecommons.org/licenses/by-nc-nd/4.0/), which permits non-commercial reuse, distribution, and reproduction in any medium, provided the original work is not changed in any way and is properly cited. For permission for commercial reuse, please email: oa@electrochem.org. [DOI: 10.1149/2.0931412jes] All rights reserved.
\end{abstract}

Manuscript submitted July 28, 2014; revised manuscript received August 29, 2014. Published September 11, 2014; Publisher error corrected September 15, 2014.

In the last decade, much attention has been drawn toward nanostructured electrodes for solid oxide fuel cells (SOFCs) prepared via infiltration techniques. ${ }^{1-7}$ The main reason is reduced polarization resistance, with corresponding increased power density, in comparison with conventional electrodes prepared via traditional mixing and sintering processes. The improved performance of infiltrated electrodes holds even at intermediate temperatures $\left(500-800^{\circ} \mathrm{C}\right)$, as demonstrated by a large number of experimental studies. ${ }^{8-13}$ Other technological advantages are a wide choice of catalyst materials, a good adhesion and a reduced thermal expansion mismatch between the electrode and the electrolyte. ${ }^{3}$ On the other hand, the long-term stability and the fabrication cost of nanostructured electrodes still need to be properly addressed. ${ }^{2,3}$

The infiltration (or impregnation) technique involves the deposition of nanoparticles into a pre-sintered backbone of micrometer-size particles. ${ }^{1,3}$ The backbone is a skeletal structure typically composed of a single component, such as yttria-stabilized zirconia (YSZ) ${ }^{5,8}$ or, in alternative, a mixed ionic-electronic conductor (e.g., lanthanum strontium cobalt ferrite LSCF). ${ }^{14}$ However, composite backbones, such as the usual lanthanum strontium manganite (LSM)/YSZ cathode, have also been used. ${ }^{1,15}$ The sintering of the backbone is performed at high temperature in order to ensure structural stability of the electrode and good connection among backbone particles. ${ }^{3}$ In the following step, nanoparticles are infiltrated in the backbone using different methods (such as metal salt precipitation, ${ }^{2}$ nanoparticle dispersion, ${ }^{16}$ molten salts $)^{1}$ and then sintered at a much lower temperature than is required in the traditional ceramic fabrication process. ${ }^{3}$ Usually several infiltration steps are repeated in order to reach the adequate amount of nanoparticles. ${ }^{1-3,6,17}$

The high electrochemical activity of infiltrated electrodes is usually attributed to the comparatively better microstructural properties exhibited by this kind of electrodes as compared to traditional electrodes. ${ }^{1,9}$ Generally speaking, the main requirements for an SOFC electrode are sufficient gas porosity, high electronic and ionic conductivity, and high activity for the electrochemical reaction. ${ }^{18}$ An infiltrated electrode possesses all these characteristics. As an example, infiltrating electron-conducting nanoparticles into an ionic-conducting backbone

*Electrochemical Society Active Member.

${ }^{\mathrm{z}}$ E-mail: antonio.bertei@for.unipi.it leads to a tremendous increase in triple-phase boundary (TPB) length as a consequence of the large number of contacts between the two different conducting phases. ${ }^{19,20}$ In addition, the backbone itself ensures a high ionic conductivity ${ }^{3}$ while a sufficient electronic conductivity is achieved provided that a connected network of nanoparticles is formed. ${ }^{4}$

Despite the important role played by the electrode microstructural characteristics, so far relatively few studies have concentrated on modeling the morphology of infiltrated electrodes. A first approach was proposed by Shah et al., ${ }^{21}$ who later extended the model into the Simple Infiltrated Microstructure Polarization Loss Estimation (SIMPLE) model. ${ }^{22,23}$ In this approach, the electrode microstructure is represented as in the Tanner-Fung-Virkar model, ${ }^{24}$ which considers the backbone structure as a series of fins covered by hemispherical nanoparticles. Obviously, such an approach provides only a geometric analogy of the real electrode microstructure and misses to predict some important features, such as the percolation threshold required to form a connected network of nanoparticles. A similar approach was adopted by Küngas et al., ${ }^{25}$ who assumed the presence of a dense film of the infiltrated phase on the backbone fins.

More descriptive models have been proposed making use of percolation theory concepts. Ding et al. ${ }^{19,26}$ presented a micro-model to predict the TPB length of electrolyte-coated anodes by calculating the number of contacts among backbone and infiltrated particles, both assumed to be spherical. Such an approach was later adopted by Zhang and $\mathrm{Xia},{ }^{27}$ who proposed a particle-layer model. A more rigorous approach, which also considers the percolation fraction of infiltrated particles, was proposed by Hardjo et al., ${ }^{28,29}$ who modeled the infiltrated phase as a film of overlapping circular disks, arranged on a $2 \mathrm{D}$ square lattice on the surface of the backbone particles. By using this approach, not only the TPB length but also the effective conductivity of the infiltrated phase was predicted. A percolation model aimed to estimate the effective electric conductivity and TPB length was also proposed by Chen et al., ${ }^{30-32}$ who considered the infiltrated electrode as a random packing of "super-particles" consisting of a core ionic-conducting backbone particle surrounded by a shell of infiltrated particles.

More recently, numerical methods which reconstruct the microstructure of infiltrated electrodes through packing algorithms have been developed in order to provide more detailed information than percolation theory models. Synodis et al. ${ }^{33}$ proposed an algorithm to 
numerically generate 3D geometries consisting of hexagonally packed unit cells representing backbone, infiltrated and pore-former particles. While using a lattice allows a fast computation, concerns about the validity of some results have been raised by Hardjo et al., ${ }^{28}$ who commented on the dependency of the specific surface area of backbone particles as a function of porosity. Zhang et al. ${ }^{34}$ presented a particlebased model to numerically reconstruct the infiltrated electrode microstructure. In this approach, after the generation and sintering of the backbone, a numerical infiltration algorithm randomly deposits nanoparticles on the surface of previously placed particles. A risk aggregation factor takes into account the tendency of nanoparticles to coat the backbone surface or to form aggregates of infiltrated particles. Although the approach is extremely powerful, the small domain size adopted and the discretization of the geometry into cubic voxels may negatively affect the accuracy of the presented results.

In this study, a particle-based modeling framework for the numerical microstructural reconstruction of infiltrated electrodes and the prediction of the effective properties is presented. Although the model shows some similarities with the Zhang et al. ${ }^{34}$ algorithm, it does not require any domain discretization to compute the TPB length and the surface area of infiltrated particles. In addition, a larger domain size is used as a result of a domain sensitivity analysis. The effective conductivity of backbone and infiltrated particles along with the effective diffusivity in gas phase are calculated and reported in dimensionless form to provide generality in their application. The study represents the extension of a previous work, ${ }^{35}$ with additional results regarding the dependency of effective properties on the contact angle among nanoparticles and the backbone porosity.

\section{Modeling}

The particle-based model reconstructs the microstructure of infiltrated electrodes in two steps: first by generating the backbone structure and then by randomly infiltrating nanoparticles. In this way, the general preparation route adopted experimentally is schematically reproduced, although not all the processing details are incorporated in the model.

Both backbone and infiltrated particles are assumed to be spherical or hemispherical, which is a simplification commonly adopted in SOFC modeling ${ }^{23,34}$ which is supported by experimental evidence. ${ }^{36-38}$ The microstructural characteristics (e.g., porosity, volume fractions) and effective properties (e.g., TPB length, effective conductivity, effective diffusivity) of the reconstructed electrodes can be calculated both before and after the infiltration.

The packing algorithms run independently of the nature of the conducting particles, which can be electron-conducting, ion-conducting, mixed ionic-electronic conducting or even inert particles. The conduction properties of the particles are only taken into account during the evaluation of effective properties. In this study, it is assumed that backbone and infiltrated particles belong to different phases, for example ion-conducting phase for backbone particles and electron-conducting phase for infiltrated particles (although also the opposite is applicable). The case of mixed ionic-electronic conducting particles is not explicitly considered in this study.

Backbone generation. - In the manufacturing process of infiltrated electrodes, the backbone is prepared by using the traditional fabrication processes adopted for conventional electrodes. As a consequence, the backbone generation is performed with the same algorithms adopted for the numerical reconstruction of conventional electrodes, which simulate the electrode microstructure as a random packing of overlapping spherical particles. ${ }^{39-43}$ In this study, the backbone is generated through a packing algorithm known as a drop-androll algorithm. ${ }^{44,45}$ Full details of the implementation and algorithm validation are reported in previous studies ${ }^{46,47}$ and a brief description of the method is summarized here.

In the drop-and-roll algorithm, backbone particles are added one at a time into a cubic box of specified dimensions by sequentially dropping spheres from a random location at the top of the domain. The incoming particle moves downward, dropping and possibly rolling over one or two already placed particles without friction or inertia. The motion stops when a stable position on the top of three particles or on the floor is reached. The procedure is repeated by adding new particles until the domain is completely filled. Wall and floor effects are removed by adopting periodic boundary conditions in the horizontal directions and by removing a layer of six particle diameters from both the top and the bottom of the domain. ${ }^{44,48}$ Although this study considers mono-component, monosized backbones, the algorithm can simulate binary mixtures and any desired particle size distribution by enforcing a weighted probability to particle selection.

The effects of the sintering of the backbone are taken into account through the adoption of a contact angle among backbone particles and the simulation of pore-former particles. The contact angle between backbone particles $\theta_{b b}$ is used to tailor the particle overlap and it is kept constant throughout the structure during the backbone generation. Pore-formers are represented in the algorithm as spherical particles, which are dropped during the backbone generation. ${ }^{40,41,49}$ Pore-former particles vanish in the final structure, thus their volume fraction determines the backbone porosity before infiltration. The algorithm also checks, after the removal of pore-former particles, if there are unstable backbone particles. ${ }^{46}$ In all the structures generated in this study, the stability criterion is met.

Random infiltration. - The infiltration of nanoparticles into the backbone microstructure is performed by using a Monte Carlo algorithm, which sequentially places nanoparticles onto the surface of backbone particles and on previously placed infiltrated particles.

The algorithm starts by randomly selecting a reference backbone particle and a random position on its surface is selected using the Marsaglia method. ${ }^{50}$ A nanoparticle is placed on the backbone particle surface at a distance corresponding to the prescribed contact angle between infiltrated and backbone particles $\theta_{i b}$ (see Figure 1). Then, the overlaps and contacts made by the nanoparticle with other backbone and infiltrated particles are evaluated by checking the center distances. A particle which touches the considered nanoparticle is marked as overlapping if the contact angle formed is larger than the desired contact angle (in particular, larger than $\theta_{i b}$ in case of an infiltratedbackbone pair, larger than $\theta_{i i}$ in case of an infiltrated-infiltrated pair, as represented in Figure 1), otherwise the touching particle is marked as a contact. By definition, the reference backbone particle on which the incoming nanoparticle is placed represents a contact.

If the considered nanoparticle does not make any overlap, its position is accepted. On the other hand, if the nanoparticle has at least one overlap there are three possibilities:

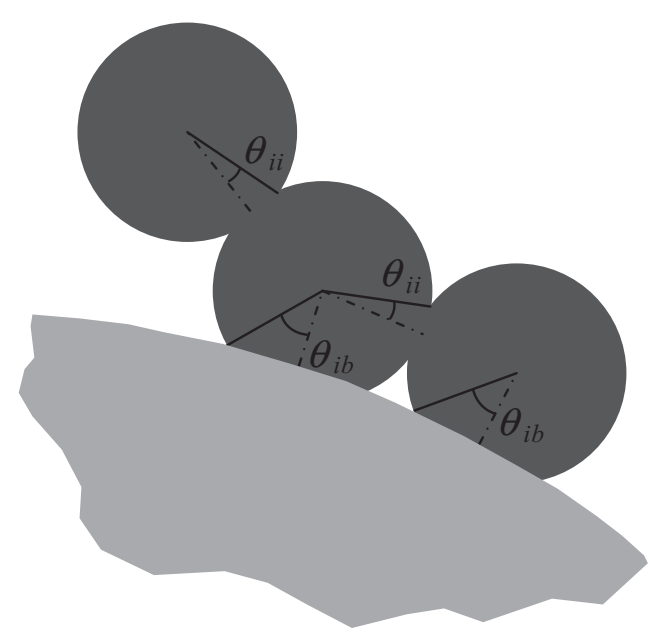

Figure 1. Definition of contact angles between infiltrated particles and backbone particles $\left(\theta_{i b}\right)$ and among infiltrated particles $\left(\theta_{i i}\right)$. 
a)

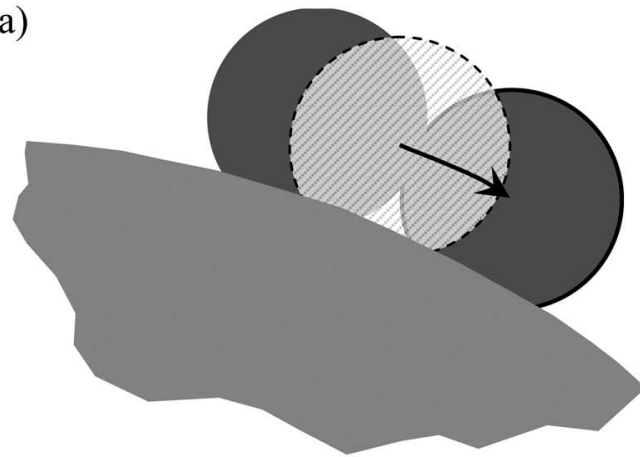

b)

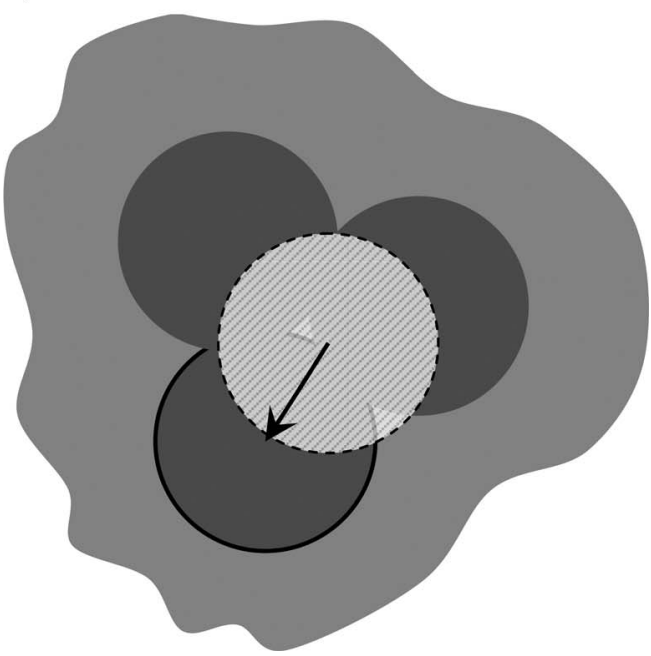

Figure 2. Schematic representation of how a nanoparticle is repositioned in the case of a) an overlap and a contact (rotation on the plane identified by particle centers) and b) two overlaps and a contact or an overlap and two contacts. In both the cases, a backbone particle represents a contact. The considered nanoparticle is identified with a dotted contour before the movement and a solid contour after the movement.

1. if the nanoparticle has one overlap and a contact with the reference backbone particle, it is repositioned over the surface of the reference backbone particle in order to remove the overlap. This is done by applying a rotation on the plane identified by the centers of the reference backbone particle, the overlapping particle and the considered nanoparticle, as represented in Figure 2a;

2 . if the number of contacts plus overlaps is three (that is, the nanoparticle has a contact and two overlaps, or two contacts and an overlap), the nanoparticle is repositioned in order to satisfy the following condition:

$$
\left|\underline{x}_{i}-\underline{x}_{j}\right|=h_{i j} \quad \text { for } j=1,2,3
$$

where $x_{i}$ is the center coordinates of the considered nanoparticle and $x_{j}$ represents the center coordinates of the three touching particles. $h_{i j}$ is the desired distance among the considered nanoparticle and the touching particle, which is calculated according to the corresponding contact angle $\left(\theta_{i b}\right.$ for infiltrated-backbone pair, $\theta_{i i}$ for infiltrated-infiltrated pair). The system of nonlinear algebraic equations in Eq. 1 is solved for $x_{i}$ through a Newton-Raphson method, ${ }^{51}$ thus providing the position of the nanoparticle which satisfies the criterion, as represented in Figure 2b;

3 . if the number of contacts plus overlaps exceeds three, the position is rejected and a new reference backbone particle is selected.

After each repositioning (i.e., cases 1 and 2), contacts and overlaps are checked again, and the nanoparticle is accepted, repositioned or rejected according to the three criteria listed above.
When a position is accepted, the whole procedure is repeated with a new nanoparticle. On the other hand, if a nanoparticle is rejected, the same nanoparticle is tested in another location by randomly selecting a new reference backbone particle. If after a prescribed number of consecutive rejections (1000 in this study) the nanoparticle has not been accepted yet, it means that the backbone surface cannot host more nanoparticles: this situation represents the monolayer limit. In this case, the algorithm continues to deposit nanoparticles onto the surface of the previously placed infiltrated particles, that is, on the first layer of infiltrated particles. This is done by randomly selecting a nanoparticle located in the first layer and by placing a new nanoparticle to a distance corresponding to the contact angle $\theta_{i i}$ with a random orientation (see Figure 1). The deposition continues until the first layer of nanoparticles is covered by a second layer, after that nanoparticles are deposited onto the second layer, and so on.

The algorithm stops to place nanoparticles when either the desired volume fraction of nanoparticles has been reached or there is no more possibility to add nanoparticles in the electrode.

At the end of the algorithm, the position and radius of each backbone and infiltrated particle in the packing are known. A sample of an infiltrated electrode numerically reconstructed with the algorithm presented is reported in Figure 3.

Effective properties. - The effective properties of the resulting packing are evaluated by using a combination of well-established techniques, such as geometric analysis, finite volume method and Monte Carlo random-walk method, which are also commonly used for the characterization of simulated conventional electrodes. In this Section, only a brief description of the techniques is reported, more details can be found in previous studies. ${ }^{46,47,52}$

The percolation properties of infiltrated particles are evaluated through the knowledge of the contact information of each nanoparticle, whose touching particles are identified as described before for contact and overlap detection. In this way, clusters of connected neighboring nanoparticles are identified. ${ }^{46,47,53}$ A cluster which spans from the top to the bottom of the domain is marked as percolating. The percolation fraction of infiltrated particles $\gamma_{i}$ is defined as the number fraction of nanoparticles belonging to percolating clusters. ${ }^{49}$ Note that, by construction, backbone particles belong to the

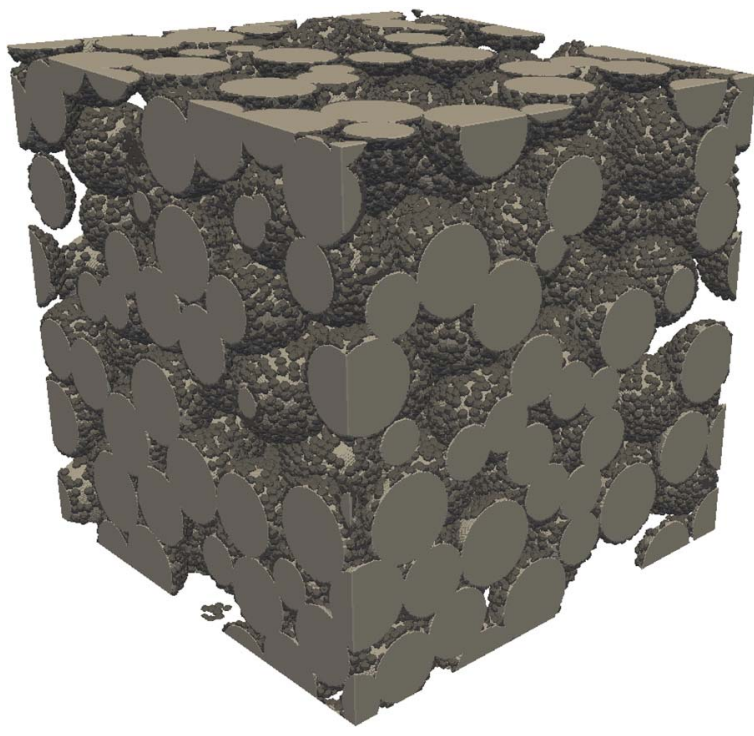

Figure 3. Infiltrated electrode generated by using the algorithms described in the Modeling Section. The backbone porosity before infiltration $\phi_{g}{ }^{B}$ is $50 \%$, the size ratio between backbone and infiltrated particles $R$ is $1 / 10$, the loading $\phi_{i}$ is 0.059 , the contact angles $\theta_{b b}=15^{\circ}, \theta_{i b}=87.1^{\circ}$ and $\theta_{i i}=30^{\circ}$. The domain, whose size $L$ is equal to six backbone particle diameters, comprises 236 backbone particles and 51567 infiltrated particles. 
same phase and are necessarily contacting, therefore their percolation fraction $\gamma_{b}$ is always 1 .

The pore percolation fraction $\gamma_{g}$ is evaluated by discretizing the domain into a Cartesian grid of cubic cells. ${ }^{39,47}$ Each cell is assigned to the gas phase if more than $50 \%$ of its volume resides in a pore. In order to ensure an accurate discretization, a cell size smaller than $1 / 10$ of the smallest nanoparticle diameter is used. ${ }^{34,54}$ Similarly to the nanoparticle percolation, the pore percolation fraction is calculated as the number fraction of cells belonging to percolating clusters.

The volume fraction $\phi$ of each phase (i.e., backbone, infiltrated and gas phase) is evaluated by randomly and uniformly distributing a large number of test points within the domain (1000 test points per backbone particle): the number fraction of points falling within a phase represents the volume fraction of that phase. ${ }^{47}$

The specific surface area of infiltrated $\left(S_{i}\right)$ and backbone particles $\left(S_{b}\right)$ exposed to gas phase is calculated by randomly generating hundreds of test points on the surface of each particle. ${ }^{55}$ The position of the test point is checked against other particles: the test point is accounted for in the calculation of the surface area if it does not belong to any other particle. For infiltrated particles, the procedure also considers if the selected particle belongs to a percolating cluster or not, in order to discriminate between the total $\left(S_{i}\right)$ and connected $\left(S_{i}{ }^{C}\right)$ specific surface area. A similar approach is used to evaluate the fraction of backbone surface exposed to the gas phase covered by infiltrated particles $\chi$.

The triple-phase boundary, which is the contact perimeter between backbone and infiltrated particles, is identified by tracking the center coordinates, the radius and the orientation of the planes of intersection among different particles. ${ }^{47}$ Hundreds of test points are generated on the perimeter of each plane of intersection connecting a backbone and an infiltrated particle. The test point is accounted for in the TPB length calculation if it does not belong to a third particle. The number fraction of accepted test points times the perimeter length of the plane of intersection represents the portion of TPB length corresponding to the considered backbone-infiltrated pair. By repeating the procedure for all the planes of intersection, the TPB length per unit volume $\lambda$ is calculated. The connected TPB length per unit volume $\lambda^{C}$ is similarly evaluated by considering infiltrated particles belonging to percolating clusters only.

The effective electric conductivity of backbone $\left(\sigma_{b}{ }^{e f f}\right)$ and infiltrated $\left(\sigma_{i}{ }^{e f f}\right)$ phase is evaluated with MicroFOAM, a finite volume method based on the open-source CFD toolkit OpenFOAM, developed and validated by Choi et al. ${ }^{52}$ for fuel cell applications. The electrode domain is discretized using body-fitted/cut-cell grids. A diffusive transport equation is solved in each conducting phase considering unit bulk conductivity in order to obtain dimensionless quantities. The evaluation of the boundary flux arising from an imposed potential difference allows the effective conductivity of the phase to be calculated.

The effective diffusivity in gas phase $D_{g}{ }^{e f f}$ is calculated by using a Monte Carlo random-walk method, whose details and validation can be found in Bertei et al. ${ }^{47}$ Thousands of tracers (more than 2000 in this study) are placed within the gas phase and allowed to move randomly following a Brownian motion in bulk regime (the Knudsen number imposed in this study is $10^{-2}$ ). At each step, tracers move up to one mean free path length and are reflected according to the Knudsen cosine law $^{56}$ when they hit the surface of a particle. After a prescribed number of random movements, equivalent to a mean displacement of five backbone particle diameters, the tracers stop. The effective diffusivity is calculated from the mean square displacement by using the Einstein equation. ${ }^{57}$ As in the case of effective conductivity, a unit bulk diffusivity is used to obtain a dimensionless effective diffusivity. ${ }^{47}$

The mean pore size is evaluated by using the chord length method: ${ }^{47}$ thousands of chords $\left(5 \cdot 10^{5}\right.$ in this study) are randomly drawn in the gas phase between two particles. The mean pore size $d_{g}$ is calculated as the number-averaged chord length $\langle l\rangle$ corrected by the statistics of chord length distribution and the nature of tracer redirecting collisions in Knudsen regime as proposed by Zalc et al. ${ }^{58}$

\section{Table I. List of parameters and corresponding ranges of variation} analyzed in this study.

\begin{tabular}{ll} 
Parameter & Range \\
\hline Electrode porosity before infiltration, $\phi_{g}{ }^{B}$ & $0.40,0.50,0.60$ \\
Size ratio, $R$ & $1 / 5,1 / 10,1 / 15,1 / 20^{\#}$ \\
Diameter backbone particles, $d_{b}$ & 1 (arbitrary units) \\
Contact angle among backbone particles, $\theta_{b b}$ & $15^{\circ}$ \\
Contact angle among infiltrated particles, $\theta_{i i}$ & $15^{\circ}, 30^{\circ}, 45^{\circ}, 60^{\circ}$ \\
Domain size, $L$ & $4,6,8,10$
\end{tabular}

${ }^{\#}$ The corresponding contact angles between infiltrated and backbone
particles $\theta_{i b}$ are $84.3^{\circ}, 87.1^{\circ}, 88.0^{\circ}, 88.5^{\circ}$.

Effective properties such as porosity, mean pore size and effective diffusivity can also be calculated for the backbone before infiltration.

\section{Results and Discussion}

The particle-based model is applied to analyze the effect of the main model parameters and electrode microstructural characteristics on the effective properties summarized in the previous Section.

The list of parameters and corresponding ranges analyzed in this study is reported in Table I. The porosity of the backbone before infiltration $\phi_{g}{ }^{B}$ is varied between $40 \%$ and $60 \%$, which is the typical range adopted for both infiltrated anodes $5,17,26,59$ and cathodes. ${ }^{6,9} \mathrm{Al}-$ though different preparation processes lead to a wide variability in the size of nanoparticles, by analyzing the SEM images reported in many experimental studies, ${ }^{3,9,26,59,60}$ the size ratio between infiltrated and backbone particles is typically on the order of $1 / 10$, which falls between the range $1 / 5-1 / 20$ investigated in this study. The contact angle among backbone particles is set to $15^{\circ}$, as commonly accepted in modeling studies on SOFC electrodes. ${ }^{28,43,49,61}$ The contact angle between infiltrated and backbone particles $\theta_{i b}$ is set in order to place nanoparticle centers exactly on the external surface of backbone particles. In this way, the first layer of infiltrated particles on the backbone surface is composed of hemispherical nanoparticles, as observed in some experimental studies ${ }^{21,36-38}$ and commonly assumed in infiltrated electrode modeling. ${ }^{22,34}$ On the other hand, there are few experimental observations of the contact angle among nanoparticles $\theta_{i i}$, therefore a wide range of $\theta_{i i}$ is considered in this study.

In order to provide generality to the data reported in this paper, all the results are given in dimensionless form by adopting a diameter of backbone particles $d_{b}$ equal to 1 in arbitrary basis. Dimensional quantities, such as the specific surface areas $\tilde{S}_{i}$ and $\tilde{S}_{b}$, the TPB length per unit volume $\tilde{\lambda}$ and the mean pore size $\tilde{d}_{g}$, can be extracted for any specific case from the dimensionless properties (i.e., $S_{i}, S_{b}, \lambda$ and $d_{g}$, respectively) by using the specific backbone diameter $\tilde{d}_{b}$ as follows: ${ }^{47,55}$

$$
\begin{gathered}
\tilde{S}_{i}=S_{i} / \tilde{d}_{b} \quad \text { and } \quad \tilde{S}_{b}=S_{b} / \tilde{d}_{b} \\
\tilde{\lambda}=\lambda / \tilde{d}_{b}^{2} \\
\tilde{d}_{g}=d_{g} \cdot \tilde{d}_{b}
\end{gathered}
$$

where the symbol $\sim$ denotes dimensional quantities. In addition, the specific effective conductivities $\left(\tilde{\sigma}_{i}^{e f f}\right.$ and $\left.\tilde{\sigma}_{b}^{e f f}\right)$ and diffusivity $\left(\tilde{D}_{g}^{e f f}\right)$ can be calculated for any specific case by multiplying the normalized values $\left(\sigma_{i}{ }^{e f f}, \sigma_{b}{ }^{e f f}\right.$ and $D_{g}{ }^{\text {eff }}$, respectively) by the bulk conductivities $\tilde{\sigma}_{i}$ and $\tilde{\sigma}_{b}$ and diffusivity $\tilde{D}_{g}$ as follows: ${ }^{47}$

$$
\begin{gathered}
\tilde{\sigma}_{i}^{e f f}=\sigma_{i}^{e f f} \cdot \tilde{\sigma}_{i} \quad \text { and } \quad \tilde{\sigma}_{b}^{e f f}=\sigma_{b}^{e f f} \cdot \tilde{\sigma}_{b} \\
\tilde{D}_{g}^{e f f}=D_{g}^{e f f} \cdot \tilde{D}_{g}
\end{gathered}
$$

Due to the randomness of the structure generation, all the numerical results are averaged over five structures per setting, according to 
Bertei et al. ${ }^{47}$ In particular, the percolation fraction $\gamma_{i}$ and related quantities, such as $\lambda^{C}$ and $S_{i}{ }^{C}$, are averaged on all the structures generated per setting, thus accounting for zero probability for non percolating realizations.

Domain size. - The main effective properties of interest in infiltrated electrodes, such as the connected TPB length or the effective conductivity of infiltrated particles, depend on the percolation properties of nanoparticles, in particular on the percolation fraction of the infiltrated phase. As known from percolation theory, ${ }^{62-64}$ the percolation properties of a packing depend on the domain size: theoretically, percolation properties are univocally defined only in an infinite domain. However, in practical applications infiltrated electrodes do not represent infinite domains. Normally the electrode thickness is on the order of tens of backbone particle diameters. It is therefore important to study how the percolation properties of infiltrated particles vary as a function of domain size.

Figure 4 shows the percolation fraction of infiltrated particles $\gamma_{i}$ and the connected TPB length per unit volume $\lambda^{C}$ as a function of the loading $\phi_{i}$ for different domain sizes $L$, which are multiples of the diameter of backbone particles. Other parameters used in the simulation are as follows: $R=1 / 10, \phi_{g}{ }^{B}=0.50, \theta_{b b}=15^{\circ}, \theta_{i i}=30^{\circ}$, $\theta_{i b}=87.1^{\circ}$.

For any domain size, both $\gamma_{i}$ and $\lambda^{C}$ increase monotonically as the loading increases. A critical loading $\phi_{i, c r}$ is identified below which the percolation fraction of infiltrated particles is zero. $\phi_{i, c r}$ represents the percolation threshold, ${ }^{28}$ that is, the minimum volume fraction at which a percolating cluster of infiltrated particles is formed. Figure $4 \mathrm{a}$ shows that the percolation threshold decreases as the domain size decreases, similar to the case of regular lattices with free boundary conditions. ${ }^{63,64}$ However, just beyond the critical loading, the percolation fraction of infiltrated particles is almost independent of the domain size since all results collapse to a single curve. In addition, the relative standard deviation of the percolation fraction $\varepsilon_{\gamma i}$ (see the graph embedded in Figure 4a) decreases as both $\phi_{i}$ and $L$ increase, being smaller than 1 only for $L \geq 6$. This indicates that a domain size $L=4$ is not big enough to guarantee adequate precision in the evaluation of effective properties, especially close to the percolation threshold. The same behavior is shown in Figure $4 \mathrm{~b}$ considering the connected TPB length per unit volume.

For an accurate evaluation of the effective properties, the results reported in Figure 4 suggest that a domain size equal to at least 6 backbone particle diameters is required. Accordingly, in the remainder of the study the domain size adopted in all the simulations is $L=6$.

Contact angle among infiltrated particles. - The effect of the contact angle among infiltrated particles $\theta_{i i}$ is reported in Figure 5 as a function of the fraction of backbone surface covered by nanoparticles $\chi$, considering $R=1 / 10, \phi_{g}{ }^{B}=0.50, \theta_{b b}=15^{\circ}, \theta_{i b}=87.1^{\circ}$. All simulations are stopped at the monolayer limit, hence there is no possibility of closed pores. As a consequence, the percolation fraction of the gas phase is always equal to 1 and there is no need to account for the gas phase percolation in either the connected TPB length $\lambda^{C}$ or the connected surface area of infiltrated particles $S_{i}{ }^{C}$ per unit volume.

Figure 5 shows that as $\theta_{i i}$ increases, the fraction of backbone surface at the monolayer limit $\chi_{m l}$ increases: $\chi_{m l}$ is equal to 0.582 , $0.677,0.805,0.961$ for $\theta_{i i}=15,30,45$ and $60^{\circ}$, respectively. As the contact angle $\theta_{i i}$ increases, infiltrated particles can overlap more. This results in a larger number of nanoparticles deposited onto the backbone surface and in an increased coverage fraction at the monolayer limit.

Figure 5a shows that, beyond the percolation threshold, the percolation fraction of infiltrated particles increases monotonically as $\chi$ increases. Interestingly, for $\theta_{i i}=15^{\circ}$ infiltrated particles do not percolate for any loading up to the monolayer limit. This is a consequence of the poor connectivity achieved with such a small contact angle, which does not allow nanoparticles to make enough contacts to create percolating clusters. Indeed, given the coverage fraction $\chi$, as $\theta_{i i}$ increases, the number of infiltrated particles increases as a consequence of the larger overlaps allowed. Consequently, the mean number of contacts
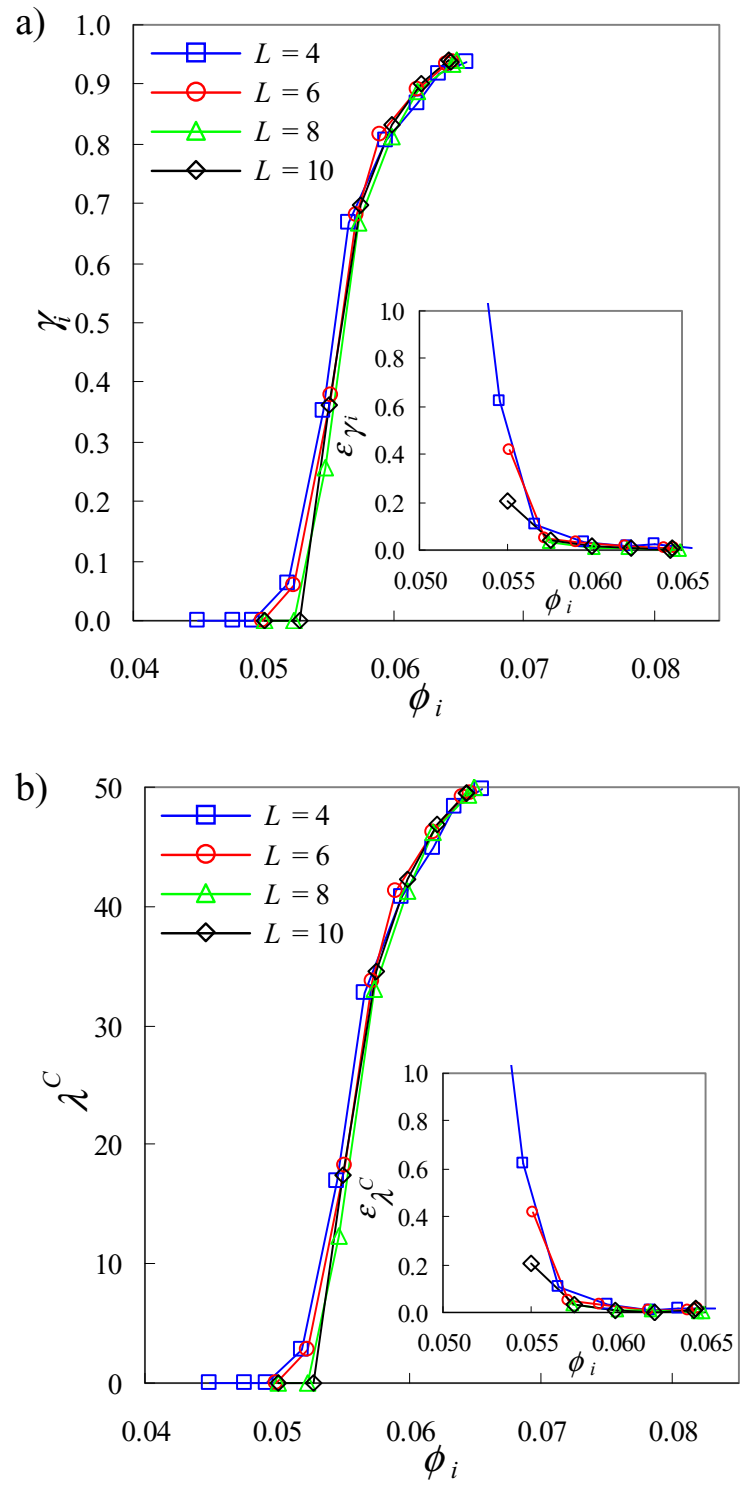

Figure 4. Effective properties for different domain sizes $L$ : a) percolation fraction of infiltrated particles $\gamma_{i}$,b) connected TPB length per unit volume $\lambda^{C}$ as a function of the loading of infiltrated particles $\phi_{i}$. The relative standard deviation $\varepsilon$ of $\gamma_{i}$ and $\lambda^{C}$ as a function of $\phi_{i}$ is embedded in the figure. Other parameters used in the simulation: size ratio $R=1 / 10$, porosity before infiltration $\phi_{g}{ }^{B}=0.50$, contact angles $\theta_{b b}=15^{\circ}, \theta_{i i}=30^{\circ}, \theta_{i b}=87.1^{\circ}$. The simulation was stopped at the monolayer limit.

among infiltrated particles increases accordingly: simulations show that at $\chi=0.582$, corresponding to the monolayer limit for $\theta_{i i}=15^{\circ}$, the mean number of contacts among infiltrated particles is $2.02,2.24$, 2.45 and 2.85 for $\theta_{i i}=15,30,45$ and $60^{\circ}$, respectively. Accordingly, the percolation threshold $\chi_{c r}$ slightly decreases as $\theta_{i i}$ increases: this is an additional consequence of the larger number of contacts created for large contact angles.

On the other hand, it is interesting to mention that infiltrated particles start to percolate even for $\theta_{i i}=15^{\circ}$ if the infiltration is continued beyond the monolayer limit (see graph embedded in Figure 5a). The percolation threshold is reached as soon as a few nanoparticles are deposited into the second layer, the critical loading $\phi_{i, c r}=0.058$ is very close to the monolayer limit $\phi_{i, m l}=0.054$. This additional loading beyond the monolayer limit is sufficient to increase the nanoparticle connectivity and thus to allow infiltrated particles to percolate.

At the percolation threshold, the mean number of contacts among infiltrated particles is $2.08,2.15,2.31$ and 2.61 for $\theta_{i i}=15,30,45$ 


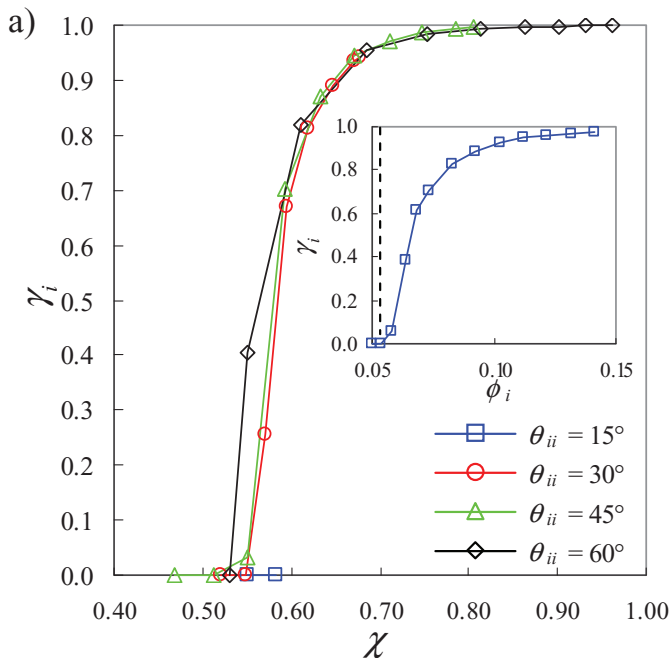

b)

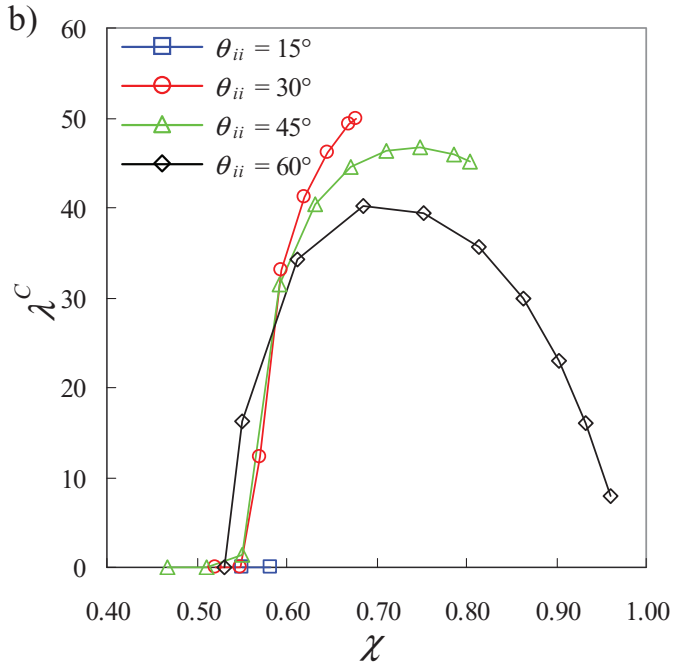

c)

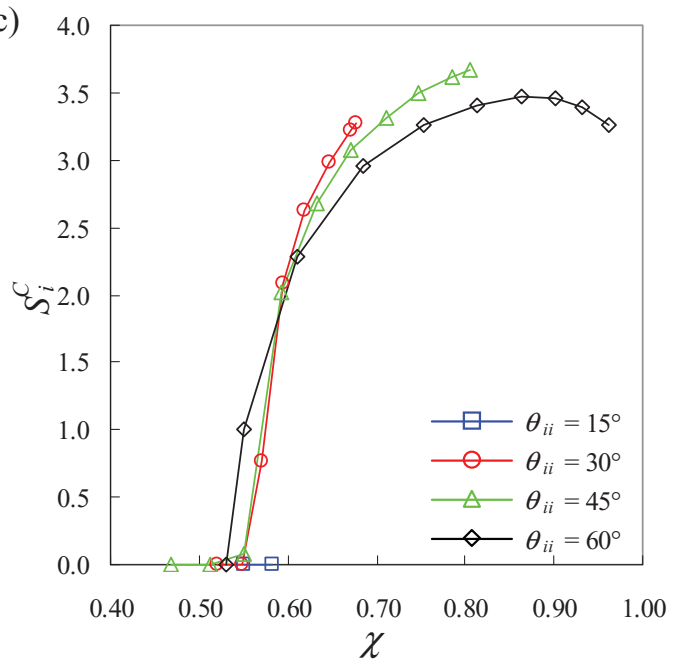

Figure 5. Effective properties for different contact angles among infiltrated particles $\theta_{i i}:$ a) percolation fraction of infiltrated particles $\gamma_{i}$, b) connected TPB length per unit volume $\left.\lambda^{C}, c\right)$ connected surface area of infiltrated particles per unit volume $S_{i}{ }^{C}$ as a function of the fraction of backbone surface covered by infiltrated particles $\chi$. Other parameters used in the simulation: size ratio $R=1 / 10$, porosity before infiltration $\phi_{g}{ }^{B}=0.50$, domain size $L=6$, contact angles $\theta_{b b}=15^{\circ}, \theta_{i b}=87.1^{\circ}$. The simulation was stopped at the monolayer limit, except for $\theta_{i i}=15^{\circ}$ in the graph embedded in Figure 5a for which the simulation was continued also beyond the monolayer limit, which is indicated with a vertical dashed line. and $60^{\circ}$, respectively. This result suggests that there is a relationship between percolation threshold, mean number of contacts among infiltrated particles and particle overlap $\theta_{i i}$. This relationship has an analogy with the percolation in random packings of spherical particles: Bouvard and Lange ${ }^{53}$ showed that the percolation threshold depends on the mean number of contacts for rigid particles, while Powell $^{65}$ and Ahmadzadeh and Simpson ${ }^{66}$ revealed that the mean number of contacts at the percolation threshold increases as the particle overlap increases. The simulation results obtained in this study indicate that the percolation properties of infiltrated electrodes obey these fundamental relations.

Figure $5 \mathrm{~b}$ shows the connected TPB length per unit volume for different contact angles up to the monolayer limit. For $\chi<0.69$, the trend of $\lambda^{C}$ as a function of $\chi$ is similar to that of the percolation fraction $\gamma_{i}$, that is, a monotonic increase in TPB length as the fraction of backbone surface covered by infiltrated particles increases. Accordingly, no connected TPB length is formed for $\theta_{i i}=15^{\circ}$, which is expected since the connected TPB length is proportional to the percolation fraction of infiltrated particles. ${ }^{28,49}$ However, for $\chi>0.69$ and $\theta_{i i} \geq 45^{\circ}$, the TPB length reaches a maximum and then decreases as the surface coverage fraction $\chi$ increases, similar to what reported by Zhang et al., ${ }^{34}$ who imposed a contact angle among infiltrated particles of $60^{\circ}$ in their numerical simulations. This decrease in connected TPB length is due to the reduction in the total TPB length per unit volume as a consequence of the larger overlaps among infiltrated particles as $\theta_{i i}$ increases. The overlaps among nanoparticles on the backbone surface reduce the contact perimeter between infiltrated and backbone particles as $\chi$ increases, thus leading to a reduction in TPB length. For the same reason, for any value of $\chi$ larger than 0.60 , the smaller the contact angle, the larger the connected TPB length.

Figure $5 \mathrm{c}$ shows the connected surface area of infiltrated particles as a function of the fraction of backbone surface covered by nanoparticles. The same considerations discussed above for the connected TPB length apply to the connected surface area of infiltrated particles, although the decrease in $S_{i}{ }^{C}$ occurs for larger $\chi$. In particular, it is interesting to note that only for $\theta_{i i}=60^{\circ}$ the maximum in $S_{i}{ }^{C}$ appears before the monolayer limit $\chi_{m l}$. By extrapolating the curve at $\chi=1$, the corresponding $S_{i}{ }^{C}(\chi \rightarrow 1)$ would be 2.94 , which is close to specific surface area of backbone particles before infiltration, equal to $S_{b}{ }^{B}$ $=2.765$. This means that, for $\theta_{i i}=60^{\circ}$, the layer of overlapped hemispherical nanoparticles behaves similarly to a film of infiltrated phase, which homogeneously covers the surface of backbone particles. This capability makes the model applicable even when the infiltration process, due to a high sintering temperature or electrode aging, leads to the formation of a film ${ }^{8,9,25,67}$ rather than a dispersion of hemispherical nanoparticles on the backbone surface.

The analysis of the results reported in Figure 5 leads to two considerations. From a modeling point of view, Figure 5 shows that, given the loading, a contact angle $\theta_{i i}=30^{\circ}$ maximizes the effective properties relevant for the electrochemical reaction, such as the TPB length and the specific surface area of infiltrated particles. Accordingly, a contact angle among infiltrated particles of $30^{\circ}$ is used in the remainder of this study in order to predict the best properties that an infiltrated electrode can provide. From a practical point of view, simulation results suggest a need to optimize the sintering process in order to ensure an adequate contact angle among infiltrated particles: not too small in order to guarantee a good connectivity, not too high in order to avoid the formation of a dense film of infiltrated phase.

Backbone porosity before infiltration.- The porosity of the backbone before infiltration $\phi_{g}{ }^{B}$ is one of the most important parameters used to modify the electrode effective properties. ${ }^{26,28,33,34}$ Experimentally, the porosity of the backbone before infiltration can be easily modified by using pore-formers and by tailoring the sintering conditions. Table II and Figure 6 report the most significant effective properties in both gas and solid phase for three different backbone porosities as a function of the loading of infiltrated particles for $R=1 / 10, \theta_{b b}=15^{\circ}, \theta_{i i}=30^{\circ}, \theta_{i b}=87.1^{\circ}$. The maximum loading 


\begin{tabular}{|c|c|c|c|}
\hline Parameter & $\phi_{i}=0$ & $\phi_{i}=0.0745$ & $\phi_{i}=0.1524$ \\
\hline$\gamma_{g}$ & 1.0 & 1.0 & 1.0 \\
\hline$d_{g}$ & 0.357 & 0.308 & 0.152 \\
\hline$D_{g}$ eff & 0.258 & 0.168 & 0.103 \\
\hline$\left\langle 1^{2}\right\rangle /\left(2 \cdot\langle 1\rangle^{2}\right)$ & 0.996 & 1.962 & 2.260 \\
\hline
\end{tabular}

analyzed in this Section is about three times the loading at the monolayer limit $\phi_{i, m l}$.

In Figure 6a, the connected TPB length per unit volume is reported: a monotonic increase in $\lambda^{C}$ as a function of the loading is shown for all the three backbone porosities investigated. Simulations reveal that the gas phase is entirely percolating in all cases, therefore, there is no decrease in connected TPB length due to percolation phenomena related to the gas phase. A reduction in gas phase percolation fraction is expected only for higher loadings and for larger contact angles among infiltrated particles, as reported by Zhang et al. ${ }^{34}$

Figure 6a shows that as the backbone porosity increases, the critical loading $\phi_{i, c r}$ at the percolation threshold decreases. This behavior is reasonable because the percolation properties of infiltrated particles depend on the backbone surface coverage fraction $\chi$ as discussed above. In particular, a critical coverage fraction $\chi_{c r}$, which is in the order $\chi_{c r}=0.52-0.54$ (see Figure $5 \mathrm{a}$ ), must be overcome to create a percolating cluster of infiltrated particles. As the backbone porosity increases, the backbone specific surface area decreases $\left(S_{b}{ }^{B}\right.$ $=3.276,2.765$ and 2.226 for $\phi_{g}{ }^{B}=0.40,0.50$ and 0.60 , respectively). Therefore, given the loading, a larger fraction of backbone surface is covered by nanoparticles as $\phi_{g}{ }^{B}$ increases, hence nanoparticle percolation occurs at a smaller loading. This same consideration is also supported by Hardjo et al., ${ }^{28}$ whose percolation model correctly predicts the percolation thresholds $\phi_{i, c r}=0.059$ and 0.050 for $\phi_{g}{ }^{B}=0.40$ and 0.50 , respectively, in the same working conditions used in this Section.

As shown in Figure 6a, for each $\phi_{g}{ }^{B}$, the connected TPB length per unit volume increases sharply after the critical loading, then $\lambda^{C}$ increases only slightly and remains almost constant as the loading increases. The first jump in TPB length is due to the increase in both total TPB length and percolation fraction of infiltrated particles as the loading increases up to the monolayer limit, similarly to that reported in Figure 4b. Once the monolayer limit is reached, corresponding to $\phi_{i, m l}=0.0745,0.0646$ and 0.0537 for $\phi_{g}{ }^{B}=0.40,0.50$ and 0.60 , additional nanoparticles are deposited in a second layer. This second layer of nanoparticles does not contribute to an increased total TPB length, it only improves the connectivity of the infiltrated phase: the percolation fraction of infiltrated particles $\gamma_{i}$ rises from 0.93 at $\phi_{i, m l}$ to 0.996 at $3 \cdot \phi_{i, m l}$ for all the three backbone porosities. Hence, the weak increase in connected TPB length after the monolayer limit is due to the slight increase in the percolation fraction of infiltrated particles. From a practical point of view, such a dependency of the connected TPB length on the loading may help researchers in optimizing the electrode microstructure. Once a monolayer of infiltrated particles is formed, which occurs at $\phi_{i, m l}$, an accurate control of the loading is not necessary since a moderate excess of nanoparticles does not lead to any detrimental reduction in connected TPB length.

Finally, Figure 6a shows that as the backbone porosity increases, the maximum TPB length per unit volume decreases. This result is reasonable because the total TPB length depends linearly on the number of backbone particles per unit volume, as suggested by percolation models. ${ }^{26,28}$ Simulations show that the number of backbone particles per unit volume decreases as $\phi_{g}{ }^{B}$ increases, thus explaining why the maximum of $\lambda^{C}$ decreases as $\phi_{g}{ }^{B}$ increases as reported in Figure 6a.
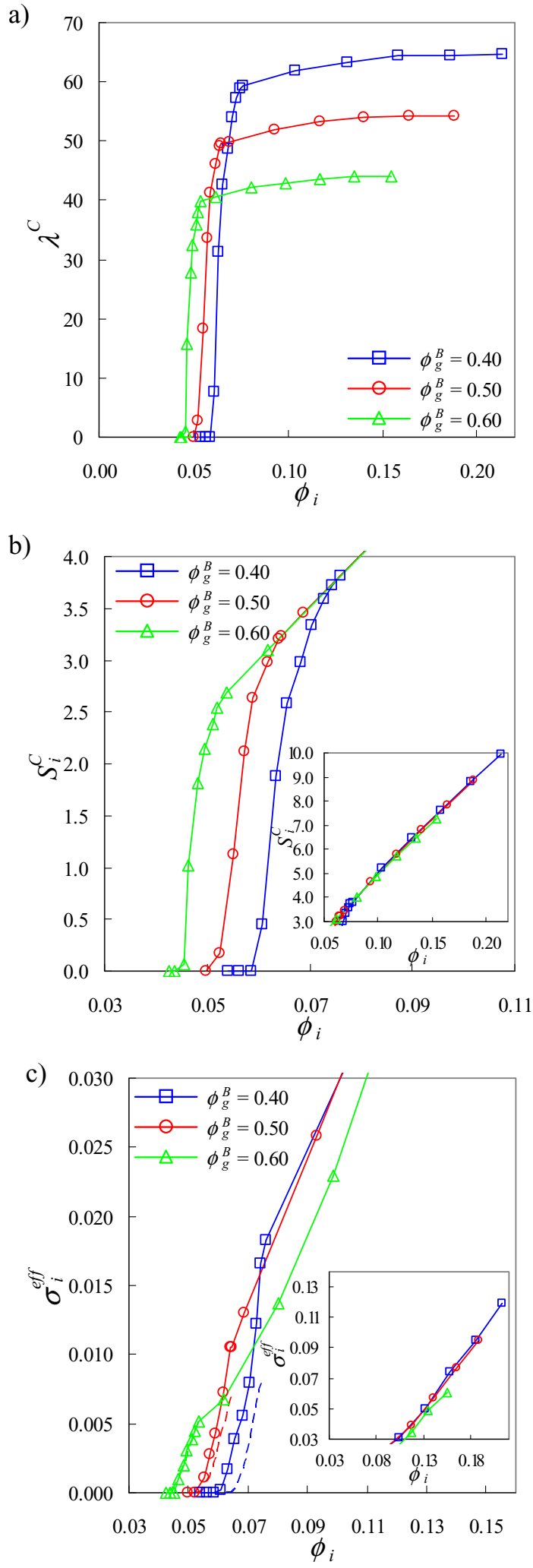

Figure 6. Effective properties for different electrode porosities before infiltration $\phi_{g}{ }^{B}:$ a) connected TPB length per unit volume $\lambda^{C}$, b) connected surface area of infiltrated particles per unit volume $S_{i}{ }^{C}$, c) effective conductivity of infiltrated particles $\sigma_{i}{ }^{e f f}$ as a function of the loading of infiltrated particles $\phi_{i}$. Other parameters used in the simulation: size ratio $R=1 / 10$, domain size $L=6$, contact angles $\theta_{b b}=15^{\circ}, \theta_{i i}=30^{\circ}, \theta_{i b}=87.1^{\circ}$. The simulation was continued also beyond the monolayer limit. The dashed lines in Figure $6 \mathrm{c}$ represent the prediction of the Chen et al. ${ }^{30}$ analytical model up to the monolayer limit. 
Figure $6 \mathrm{~b}$ shows the connected surface area of infiltrated particles per unit volume as a function of the loading for different backbone porosities. Below the monolayer limit, the dependency of $S_{i}{ }^{C}$ on $\phi_{i}$ is similar to that of the connected TPB length, as $S_{i}{ }^{C}$ increases sharply after the critical loading. However, beyond the monolayer limit the connected surface area of infiltrated particles continues to increase significantly with a constant slope for all the backbone porosities. In particular, as shown in the detail embedded in Figure 6b, all the curves collapse on each other regardless of the backbone porosity. This happens because every nanoparticle infiltrated beyond the monolayer limit contributes to an increased connected specific surface area since the percolation fraction of infiltrated particles is practically 1 as discussed above. The contribution of each additional nanoparticle to $S_{i}{ }^{C}$ is equal to the surface area of the nanoparticle minus the fraction of surface area lost in overlaps with other particles. Eventually, there is a trivial linear relationship between the incremental contribution of surface area added by each additional infiltrated particle and the volume fraction $\phi_{l}$, resulting in a linear relationship between $S_{i}{ }^{C}$ and $\phi_{\iota}$ independent of the backbone porosity.

Concluding, Figure $6 \mathrm{~b}$ suggests that a large specific connected surface area can be reached when infiltrating nanoparticles beyond the monolayer limit. This can lead to beneficial effects when the electrode electrochemical performance is affected by surface-reaction limitations. ${ }^{25}$

Figure $6 \mathrm{c}$ reports the effective conductivity of infiltrated particles per unit volume as a function of the loading. The curves of $\sigma_{i}{ }^{e f f}$ for different backbone porosities show a trend similar to those of $S_{i}{ }^{C}$ : a sharp increase in effective conductivity after the critical loading until $\phi_{i, m l}$, then eventually the curves collapse on each other keeping to increase. This behavior suggests that, for $\phi_{i}>\phi_{i, m l}, \sigma_{i}$ eff depends mainly on the disposition and volume fraction of infiltrated particles placed in the additional layers, so that the effective conductivity of infiltrated particles depends essentially on the loading rather than on the backbone porosity.

In Figure $6 \mathrm{c}$ the numerical results are cross-checked with the analytical model proposed by Chen et al. ${ }^{30}$ The theoretical model is applied up to the monolayer limit considering that the first layer is made of hemispherical nanoparticles, resulting in a multiplier 2 for the solid volume fraction of infiltrated particles in the calculation of the equivalent radius of the super-particle (i.e., the backbone particle surrounded by infiltrated particles). The apparent porosity of the layer of nanoparticles is taken equal 0.18 , that is equal to the porosity of a random packing of particles with contact angle $\theta_{i i}=30^{\circ} .{ }^{47}$ Figure $6 \mathrm{c}$ shows that numerical and theoretical results perfectly match for $\phi_{g}{ }^{B}=0.60$, while the agreement worsens for $\phi_{g}{ }^{B}=0.50$ and 0.40 . However, the comparison is still satisfactory, indicating that both the models provide results consistent each other.

It is interesting to note that the specific effective conductivity of infiltrated particles is generally smaller than the specific effective conductivity of backbone particles. For example, while $\sigma_{b}{ }^{\text {eff }}=0.211$, 0.133 and 0.073 for $\phi_{g}{ }^{B}=0.40,0.50$ and 0.60 , the corresponding specific effective conductivity of infiltrated particles at the monolayer limit is $\sigma_{i, m l} e^{e f f}=0.0166,0.0105$ and 0.0052 , that is, more than an order of magnitude smaller than $\sigma_{b}{ }^{\text {eff }}$. This result is reasonable because the conduction within infiltrated particles involves a highly tortuous and narrow conduction path on the surface of backbone particles. It must be mentioned that the predicted specific effective conductivity of backbone particles is larger than the typical effective conductivity in conventional composite electrodes, ${ }^{47}$ in agreement with experimental observations. $^{3}$

The last consideration is about effective properties in gas phase, which are summarized in Table II for the least porous structures analysed in this study.

As anticipated above, in the range of loading analyzed, the infiltration has no effect on the percolation fraction of the gas phase $\gamma_{g}$, which is constantly 1 . This is probably the main indication to support the general opinion that an adequate amount of infiltrated particles into a porous backbone should not significantly compromise the gas phase transport properties of the electrode. However, obviously the infiltration process reduces the electrode porosity as $\phi_{g}=\phi_{g}{ }^{B}-\phi_{i}$. Since nanoparticles are deposited onto the backbone surface, it is not surprising that simulations show that the mean pore size $d_{g}$ decreases as the loading increases. $d_{g}$ is reduced by a fraction of nanoparticle diameter, which is equal to $d_{i}=0.1$, as the loading increases from 0 to the monolayer limit $\phi_{i, m l}=0.0745$. On the other hand, the mean pore size is halved when the loading approaches $2 \cdot \phi_{i, m l}$, as a consequence of the formation of multiple layers of infiltrated particles on the backbone surface. It is interesting to note that, for $\phi_{i}>0$, the ratio $\left\langle l^{2}\right\rangle /\left(2 \cdot\langle l\rangle^{2}\right)$ differs from 1 , which is the value reported for isotropic random packings of spheres, indicating an exponential chord-length distribution. ${ }^{58,68-70}$ Since $\left\langle l^{2}\right\rangle /\left(2 \cdot\langle l\rangle^{2}\right)>1$ for $\phi_{i}=0.0745$ and 0.1524 , a bimodal pore size distribution is expected in infiltrated electrodes. Therefore, it would be more advisable to speak about two characteristic pore sizes: one related to the mesoporosity of the backbone and one for characterizing the nanopores embedded in the layers of infiltrated particles.

More importantly, Table II shows that the infiltration of nanoparticles reduces the effective diffusivity in gas phase $D_{g}$ eff, which is consistent with the reduction in porosity and mean pore size as discussed above. Interestingly, a good prediction of $D_{g}$ eff is obtained by using the Berson et al. ${ }^{69}$ equation $D_{g}{ }^{e f f}=1.6 \cdot \phi_{g}{ }^{2}$ Although such an equation was derived in numerically-reconstructed conventional electrodes, this study suggests that it may be applicable in first approximation even in infiltrated electrodes.

From the numerical results reported in Table II, the reduction in effective diffusivity and mean pore size is relevant but not big enough to expect a dramatic limitation in the electrode electrochemical performance. This is comfortable from a practical point of view because, as mentioned above, there is no need to carefully control the excess of loading beyond the monolayer limit, although a large excess of infiltrated particles is not recommended.

Size ratio. - According to percolation models, ${ }^{26,28}$ the size ratio between infiltrated and backbone particles $R=d_{i} / d_{b}$ is expected to play an important role in determining the percolation and effective properties of infiltrated electrodes. Figure 7 shows the effect of the size ratio on connected TPB length, percolation fraction and connected surface area of infiltrated particles as a function of the fraction of backbone surface covered by nanoparticles for $\phi_{g}{ }^{B}=0.50, \theta_{b b}=15^{\circ}$ and $\theta_{i i}=30^{\circ}$. The results can be easily plotted as a function of the loading by converting the surface coverage fraction into $\phi_{i}$ as follows:

$$
\phi_{i}=m \cdot \chi \quad \text { for } \chi \leq \chi_{m l}
$$

where $m=0.1792,0.0964,0.0659,0.0501$ for $R=1 / 5,1 / 10,1 / 15$, $1 / 20$, respectively. The linear relationship in Eq. 5 holds only up to the monolayer limit, which is the upper bound of the loading considered in this Section.

Figure $7 \mathrm{a}$ shows the percolation fraction of infiltrated particles as a function of the surface coverage fraction. As $R$ increases, the percolation threshold $\chi_{c r}$ decreases. Accordingly, given $\chi$, the percolation fraction $\gamma_{i}$ increases as $R$ increases. This percolating behavior is typical of confined systems ${ }^{64}$ and can be explained by using a simple analogy. In infiltrated electrodes, the percolation of nanoparticles must occur along a given direction (for example, the vertical one) on the surface of each backbone particle. This system shows a similarity with the percolation in a square lattice with periodic boundary conditions imposed in the horizontal direction: the lattice area represents the surface of the backbone particle flattened in $2 \mathrm{D}$, while the site occupation fraction $q$ of the lattice is the equivalent of the fraction of backbone surface occupied by infiltrated particles $\chi$. In this analogy, increasing the size of the lattice is equivalent to reducing the size ratio $R$ in infiltrated electrodes.

Figure 8 shows the percolation fraction $\gamma$ as a function of the site occupation fraction $q$ in a square lattice. Results are averaged over $10^{4}$ random realizations following the method presented by Hoshen and Kopelman. ${ }^{71}$ Figure 8 shows that it is more likely to reach percolation in a small domain rather than in a big one, at least for $q<$ 
a)

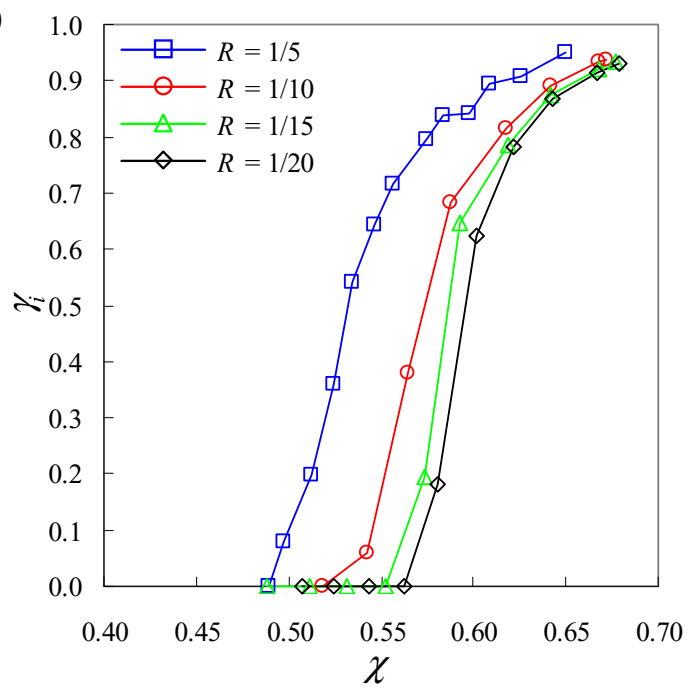

b)

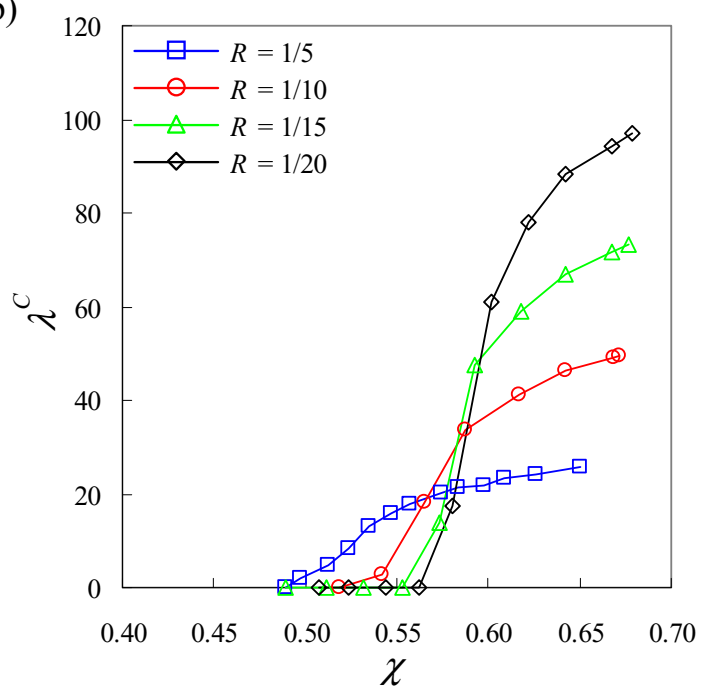

c)

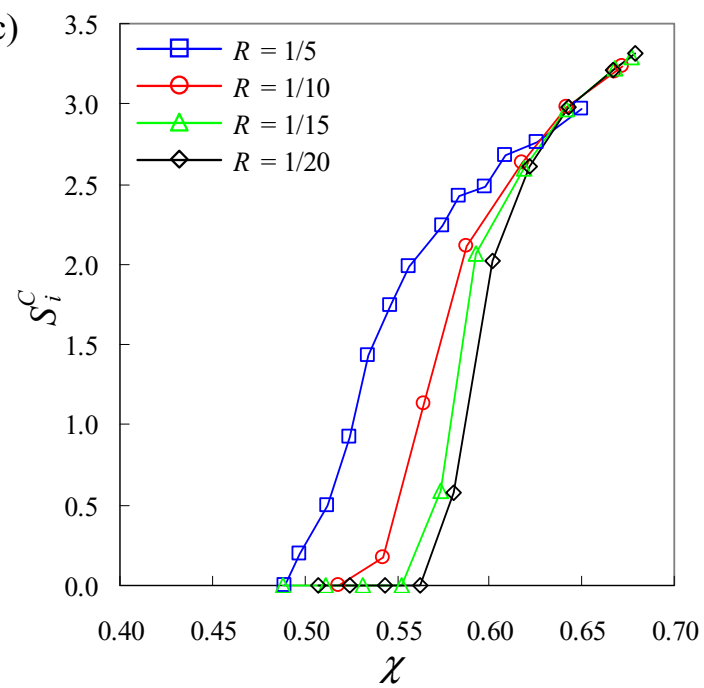

Figure 7. Effective properties for different size ratios $R$ : a) percolation fraction of infiltrated particles $\gamma_{i}$, b) connected TPB length per unit volume $\lambda^{C}$ c) connected surface area of infiltrated particles per unit volume $S_{i}{ }^{C}$ as a function of the fraction of backbone surface covered by infiltrated particles $\chi$. Other parameters used in the simulation: porosity before infiltration $\phi_{g}{ }^{B}=$ 0.50 , domain size $L=6$, contact angles $\theta_{b b}=15^{\circ}, \theta_{i i}=30^{\circ}, \theta_{i b}=84.3^{\circ}$, $87.1^{\circ}, 88.0^{\circ}, 88.5^{\circ}$ for $R=1 / 5,1 / 10,1 / 15,1 / 20$, respectively. The simulation was stopped at the monolayer limit.

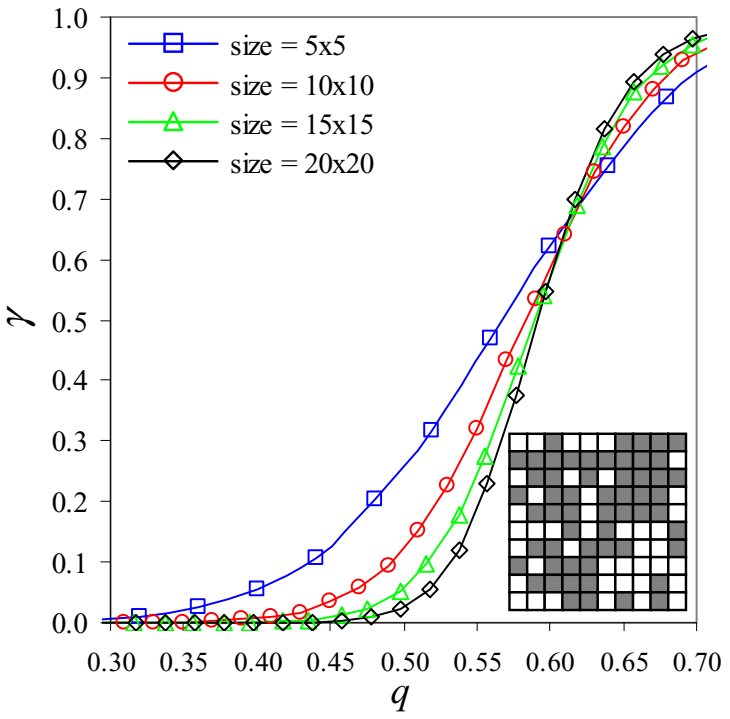

Figure 8. Percolation fraction in a square lattice for different domain sizes as a function of the site occupation fraction. Percolation is assessed along the vertical direction over $10^{4}$ random realizations, periodic boundary conditions are imposed in the horizontal direction. An example of realization (size $10 \times 10, q=0.60)$ is embedded in the figure.

0.6. The similarity between Figure 8 and Figure $7 \mathrm{a}$ is evident and explains why in infiltrated electrodes (Figure 7a) the percolation fraction is higher as $R$ increases given the surface coverage fraction $\chi$. From a different point of view, simulation results indicate that, for $R>1 / 20$, backbone particles are not big enough to extinguish finitesize percolation effects. As a consequence, the percolation of infiltrated particles is not independent of the size ratio $R$. Percolation models which neglect this phenomenon are expected to be inaccurate for the prediction of effective properties in infiltrated electrodes.

Table III summarizes the percolation thresholds obtained in this study for different size ratios $R$. As discussed above, $\chi_{c r}$ increases as $R$ decreases. However, it is important to emphasize that the percolation threshold expressed in terms of critical loading $\phi_{i, c r}$ decreases as $R$ decreases. This behavior is merely related to the fact that the smaller the size ratio $R$, the smaller the volume fraction of infiltrated particles $\phi_{i}$ given the coverage fraction $\chi$ since nanoparticles are smaller. Table III also reports the effective conductivity of infiltrated particles at the monolayer limit for different size ratios. As $R$ increases, the effective conductivity of infiltrated particles increases. This trend is reasonable because, since $\chi_{m l}$ is almost constant for different $R$, as $d_{i}$ increases the contact area among infiltrated particles, which is the bottleneck for conduction, increases.

Figure $7 \mathrm{~b}$ shows the connected TPB length per unit volume as a function of the fraction of backbone surface covered by infiltrated particles. The dependency of $\lambda^{C}$ on $\chi$ mirrors that of $\gamma_{i}$ on $\chi$ reported in Figure $7 \mathrm{a}$. However, Figure $7 \mathrm{~b}$ indicates that the TPB length scales inversely with the size ratio, i.e., with the diameter of infiltrated particles.

\begin{tabular}{|c|c|c|c|c|}
\hline Parameter & $R=1 / 5$ & $R=1 / 10$ & $R=1 / 15$ & $R=1 / 20$ \\
\hline \multicolumn{5}{|c|}{ Critical condition } \\
\hline$\chi_{c r}$ & 0.49 & 0.52 & 0.55 & 0.56 \\
\hline$\phi_{i, c r}$ & 0.088 & 0.050 & 0.036 & 0.028 \\
\hline \multicolumn{5}{|c|}{ Monolayer limit } \\
\hline$\chi_{m l}$ & 0.65 & 0.67 & 0.68 & 0.68 \\
\hline$\phi_{i, m l}$ & 0.116 & 0.065 & 0.044 & 0.034 \\
\hline$\sigma_{i, m l}^{e f f}$ & $1.56 \cdot 10^{-2}$ & $1.05 \cdot 10^{-2}$ & $0.95 \cdot 10^{-2}$ & $0.78 \cdot 10^{-2}$ \\
\hline
\end{tabular}


Such a scaling property, also predicted by percolation theory, ${ }^{28}$ is evident by comparing the values of $\lambda^{C}$ at the monolayer limit for different $R$ since, according to Figure $7 \mathrm{a}$, at $\chi=\chi_{m l}$ the percolation fraction of infiltrated particles is almost identical for all the size ratios investigated and a direct comparison can be performed.

Also the connected surface area of infiltrated particles per unit volume shows a dependency on $\chi$ similar to that of the percolation fraction $\gamma_{i}$, as reported in Figure $7 \mathrm{c}$. Interestingly, simulations show that, given the coverage fraction $\chi$, the specific surface area of infiltrated particles $S_{i}$ is independent of the size of nanoparticles. This result can be explained by considering the relationship between $S_{i}$ and $\chi$ reported as follows:

$$
S_{i} \propto \frac{S_{b}^{B} \cdot \chi}{\frac{\pi}{4} d_{i}^{2}} \cdot \frac{\pi}{2} d_{i}^{2} \propto 2 \cdot S_{b}^{B} \cdot \chi
$$

In Eq. 6, the first term in central part of the equation represents the number of infiltrated particles per unit volume, which is proportional to the surface area of backbone particle covered by nanoparticles $\left(S_{b}{ }^{B} \cdot \chi\right)$ divided by the area occupied by each infiltrated particle, that is, its cross section $\pi / 4 \cdot d_{i}{ }^{2}$. The specific surface area of infiltrated particles $S_{i}$ is equal to the number of infiltrated particles per unit volume times the surface area exposed by each infiltrated particle, which is proportional to $\pi / 2 \cdot d_{i}{ }^{2}$. Eventually, as reported in the rightmost side of Eq. 6, $S_{i}$ is independent of the diameter of nanoparticles and so on the size ratio $R$ given the surface coverage fraction $\chi$.

As a final comment, simulations show that most of the effective properties, such as the connected TPB length $\lambda^{C}$, the effective conductivity $\sigma_{i}{ }^{e f f}$ and the critical loading $\phi_{i, c r}$, are highly sensitive to the size ratio $R$, that is, on the size of infiltrated particles. This means that an accurate control of the size of nanoparticles is required during the preparation of infiltrated electrodes in order to obtain the desired microstructure predicted by the model. As a consequence, this feature may make the optimization of infiltrated electrode very demanding from a practical point of view.

\section{Conclusions}

The paper presented a modeling framework, based on randomsequential-addition packing algorithms, for the particle-based reconstruction of SOFC infiltrated electrodes and the prediction of their effective properties, which were reported in dimensionless form to provide generality in their application.

Simulations showed that the percolation properties of the infiltrated phase are mainly determined by the fraction of backbone surface covered by infiltrated particles. The critical coverage fraction was identified in the range $0.50-0.55$. In terms of volume fractions, the critical loading was found to increase as the backbone porosity decreases and the size of nanoparticles increases.

The contact angle among infiltrated particles affects the surface coverage fraction and the nanoparticle connectivity, thus influencing the percolation properties. For contact angles larger than $45^{\circ}$ nanoparticles cover an excessive fraction of backbone surface, resulting in a decrease in both TPB length and specific surface area of nanoparticles. The optimum degree of nanoparticle overlap was indentified in a contact angle of $30^{\circ}$, which should be experimentally guaranteed by tailoring the sintering conditions.

Simulations suggested that a careful control of the excess of loading is not necessary from a practical point of view because the connected TPB length became almost flat while both the specific surface area and the effective conductivity of infiltrated particles increased beyond the monolayer limit. As a rule of thumb, a loading in the order of twice the critical loading could be a reasonable compromise between TPB length, conduction and gas transport properties. On the other hand, simulations indicated that effective properties are highly sensitive to the nanoparticle size, which may make electrode optimization demanding unless a proper control of the nanoparticle size is adopted.

Concluding, the capability of the presented modeling framework to predict effective properties in a wide range of conditions makes it a valid tool to guide researchers in optimizing the electrode microstructure. Future investigations will be dedicated to study the effect of the particle size distribution, mixed ionic-electronic conducting materials and to the comparison with dedicated experimental data.

\section{List of Symbols}

$d_{1}$

$d_{g}$

$d_{g}$
$d_{i}$
$D_{g}$

$D_{g}{ }^{e f f}$

$h_{i j}$

$\langle l\rangle$

$\left\langle l^{2}\right\rangle$

$L$

$m$

$q$

$R$

$S_{b}$

$S_{i}$

$\underline{x}$

diameter of backbone particles (set to 1 in arbitrary units)

mean pore size

diameter of infiltrated particles

effective gas diffusivity (relative to a unitary bulk diffusivity)

distance between particle $i$ and particle $j$ centers (see Eq. 1)

number-averaged chord length

mean-square chord length

domain side length

parameter defined in Eq. 5

occupation probability in a square lattice

size ratio $d_{i} / d_{b}$

surface area of backbone particles per unit volume surface area of infiltrated particles per unit volume coordinates of particle center

\section{Superscripts}

$B$

$C$

before infiltration

connected (i.e., corresponding to percolating infiltrated particles)

dimensional quantity

\section{Subscripts}

$b$

cr

$g$

$i$

$m l$

$\gamma$

$\varepsilon$

$\theta_{b b}$

$\theta_{i b}$

$\theta_{i i}$

$\lambda$
$\sigma^{e f f}$

$\sigma^{e f f}$

$\phi_{b}$

$\phi_{g}$

$\phi_{i}$

$x$ backbone particles

critical (i.e., at the percolation threshold)

gas phase

infiltrated particles (i.e., nanoparticles)

monolayer limit

\section{Greek Letters}

percolation fraction

relative standard deviation

contact angle among backbone particles

contact angle among infiltrated and backbone particles

contact angle among infiltrated particles

triple-phase boundary length per unit volume

effective conductivity (relative to unitary bulk conductivity)

volume fraction of backbone particles

porosity

volume fraction of infiltrated particles

fraction of backbone surface area covered by infiltrated particles

\section{References}

1. T. Z. Sholklapper, C. P. Jacobson, S. J. Visco, and L. C. De Jonghe, Fuel Cells, 8, 303 (2008).

2. S. P. Jiang, Mater. Sci. Eng. A, 418, 199 (2006).

3. Z. Jiang, C. Xia, and F. Chen, Electrochim. Acta, 55, 3595 (2010).

4. T. Z. Sholklapper, H. Kurokawa, C. P. Jacobson, S. J. Visco, and L. C. De Jonghe, Nanoletters, 7, 2136 (2007)

5. A. N. Busawon, D. Sarantaridis, and A. Atkinson, Electrochem. Solid-State Lett., 11, B186 (2008).

6. J. M. Vohs and R. J. Gorte, Adv. Mater, 21, 943 (2009).

7. R. J. Gorte and J. M. Vohs, Curr. Opin. Colloid Interface Sci., 14, 236 (2009).

8. Y. Huang, J. M. Vohs, and R. J. Gorte, J. Electrochem. Soc., 152, A1347 (2005).

9. F. Bidrawn, G. Kim, N. Aramrueang, J. M. Vohs, and R. J. Gorte, J. Power Sources, 195, 720 (2010) 
10. X. Xu, Z. Jiang, X. Fan, and C. Xia, Solid State Ionics, 177, 2113 (2006).

11. S. P. Jiang and W. Wang, J. Electrochem. Soc., 152, A1398 (2005).

12. M. Shah and S. A. Barnett, Solid State Ionics, 179, 2059 (2008).

13. B. Mirfakhraei, S. Paulson, V. Thangadurai, and V. Birss, J. Power Sources, 243, 95 (2013).

14. M. Sahibzada, S. J. Benson, R. A. Rudkin, and J. A. Kilner, Solid State Ionics, 113, 285 (1998).

15. C. Lu, T. Z. Sholklapper, C. P. Jacobson, S. J. Visco, and L. C. De Jonghe, J. Electrochem. Soc., 153, A1115 (2006).

16. Y. Huang, J. M. Vohs, and R. J. Gorte, Electrochem. Solid-State Lett., 9, A237 (2006).

17. P. Keyvanfar and V. Birss, ECS Trans., 57, 1627 (2013).

18. M. Mogensen and S. Skaarup, Solid State Ionics, 86-88, 1151 (1996)

19. W. Zhu, D. Ding, and C. Xia, Electrochem. Solid-State Lett., 11, B83 (2008).

20. M. Kishimoto, M. Lomberg, E. Ruiz-Trejo, and N. P. Brandon, J. Power Sources, 266, 291 (2014).

21. M. Shah, J. D. Nicholas, and S. A. Barnett, Electrochem. commun., 11, 2 (2009).

22. J. D. Nicholas and S. A. Barnett, J. Electrochem. Soc., 157, B536 (2010)

23. J. D. Nicholas, L. Wang, A. V. Call, and S. A. Barnett, Phys. Chem. Chem. Phys., 14, 15379 (2012)

24. C. W. Tanner, K.-Z. Fung, and A. V. Virkar, J. Electrochem. Soc., 144, 21 (1997).

25. R. Küngas, F. Bidrawn, E. Mahmoud, J. M. Vohs, and R. J. Gorte, Solid State Ionics, 225, 146 (2012).

26. D. Ding, W. Zhu, J. Gao, and C. Xia, J. Power Sources, 179, 177 (2008).

27. Y. Zhang and C. Xia, J. Power Sources, 195, 4206 (2010).

28. E. F. Hardjo, D. S. Monder, and K. Karan, J. Electrochem. Soc., 161, F83 (2014).

29. E. Hardjo, D. S. Monder, and K. Karan, ECS Trans., 35, 1823 (2011).

30. M. Chen, T. Liu, and Z. Lin, ECS Electrochem. Lett., 2, F82 (2013).

31. M. Chen and Z. Lin, Int. J. Hydrogen Energy (2014).

32. M. Chen, C. Song, and Z. Lin, Int. J. Hydrogen Energy, 39, 13763 (2014).

33. M. J. Synodis et al., J. Electrochem. Soc., 160, F1216 (2013).

34. Y. Zhang, Q. Sun, C. Xia, and M. Ni, J. Electrochem. Soc., 160, F278 (2013).

35. A. Bertei, J. G. Pharoah, D. A. W. Gawel, and C. Nicolella, ECS Trans., 57, 2527 (2013).

36. F. Liang et al., Electrochem. commun., 11, 1048 (2009).

37. Z. Zhan, D. M. Bierschenk, J. S. Cronin, and S. A. Barnett, Energy Environ. Sci., 4 3951 (2011).

38. F. Liang et al., Int. J. Hydrogen Energy, 36, 7670 (2011).

39. B. Kenney, M. Valdmanis, C. Baker, J. G. Pharoah, and K. Karan, J. Power Sources, 189, 1051 (2009).
40. Q. Cai, C. S. Adjiman, and N. P. Brandon, Electrochim. Acta 56, 10809 (2011).

41. Y. Nishida and S. Itoh, Electrochim. Acta, 56, 2792 (2011).

42. A. Abbaspour, J.-L. Luo, and K. Nandakumar, Electrochim. Acta, 55, 3944 (2010).

43. J. Sanyal, G. M. Goldin, H. Zhu, and R. J. Kee, J. Power Sources, 195, 6671 (2010).

44. W. M. Visscher and M. Bolsterli, Nature, 239, 504 (1972).

45. E. M. Tory, B. H. Church, M. K. Tam, and M. Ratner, Can. J. Chem. Eng., 51, 484 (1973).

46. A. Bertei, H.-W. Choi, J. G. Pharoah, and C. Nicolella, Powder Technol., 231, 44 (2012).

47. A. Bertei, B. Nucci, and C. Nicolella, Chem. Eng. Sci., 101, 175 (2013).

48. R. Ben Aïm and P. Le Goff, Powder Technol., 1, 281 (1968).

49. A. Bertei and C. Nicolella, J. Power Sources, 196, 9429 (2011).

50. G. Marsaglia, Ann. Math. Stat., 43, 645 (1972).

51. T. J. Ypma, SIAM Rev., 37, 531 (1995).

52. H.-W. Choi, A. Berson, J. G. Pharoah, and S. B. Beale, Proc. Inst. Mech. Eng. Part A J. Power Energy, 225, 183 (2011).

53. D. Bouvard and F. F. Lange, Acta Metall. Mater, 39, 3083 (1991).

54. Q. Cai, C. S. Adjiman, and N. P. Brandon, Electrochim. Acta, 56, 5804 (2011).

55. A. Bertei, B. Nucci, and C. Nicolella, Chem. Eng. Trans., 32, 1531 (2013).

56. J. Greenwood, Vacuum, 67, 217 (2002).

57. A. Einstein, Investigations on the theory of the Brownian movement, Dover, New York, (1926).

58. J. M. Zalc, S. C. Reyes, and E. Iglesia, Chem. Eng. Sci., 59, 2947 (2004).

59. T. Klemensø, K. Thydén, M. Chen, and H.-J. Wang, J. Power Sources, 195, 7295 (2010).

60. T. Z. Sholklapper, C. Lu, C. P. Jacobson, S. J. Visco, and L. C. De Jonghe, Electrochem. Solid-State Lett., 9, A376 (2006).

61. P. Costamagna, P. Costa, and V. Antonucci, Electrochim. Acta, 43, 375 (1998)

62. D. Stauffer and A. Aharony, Introduction to percolation theory, Taylor \& Francis, London, (1992).

63. M. S. Watanabe, Phys. Rev. E, 51, 3945 (1995)

64. P. H. L. Martins and J. A. Plascak, Phys. Rev. E, 67, 046119 (2003).

65. M. J. Powell, Phys. Rev. B, 21, 3725 (1980).

66. M. Ahmadzadeh and A. W. Simpson, Phys. Rev. B, 25, 4633 (1982).

67. W. Wang, M. D. Gross, J. M. Vohs, and R. J. Gorte, J. Electrochem. Soc., 154, B439 (2007).

68. B. Lu and S. Torquato, J. Chem. Phys., 98, 6472 (1993).

69. A. Berson, H.-W. Choi, and J. G. Pharoah, Phys. Rev. E, 83, 026310 (2011)

70. P. Levitz, J. Phys. Chem., 97, 3813 (1993).

71. J. Hoshen and R. Kopelman, Phys. Rev. B, 14, 3438 (1976). 